\title{
Special Section Guest Editorial: Three-Dimensional Image Reconstruction in Radiology and Nuclear Medicine
}

\author{
Scott D. Metzler, ${ }^{\mathrm{a}, *}$ Samuel Matej, ${ }^{\mathrm{a}, *}$ and J. Webster Stayman ${ }^{\mathrm{b}, *}$ \\ ${ }^{a}$ The University of Pennsylvania, Department of Radiology, Philadelphia, Pennsylvania, \\ United States \\ ${ }^{\mathrm{b}}$ The Johns Hopkins School of Medicine, Department of Biomedical Engineering, \\ Baltimore, Maryland, United States
}

The International Meeting on Fully Three-Dimensional Image Reconstruction in Radiology and Nuclear Medicine is a workshop-style conference focusing on algorithmic and computational methods for reconstruction of multi-dimensional data sets typically acquired using x-ray computed tomography (CT), positron emission tomography (PET), or single photon emission computed tomography (SPECT). It is well known for bringing together experts in the field in an informal setting for greater depth of discussion on issues pertaining to reconstruction. The fact that nearly all recent major advances in image reconstruction have been first presented at the Fully3D meeting emphasizes the high relevance of this meeting. The $15^{\text {th }}$ biennial conference occurred in June 2019, on the campus of The University of Pennsylvania, in Philadelphia, PA.

The conference covered the traditional range of topics focused on reconstruction algorithms and approaches, as well as novel acquisition techniques and geometries for CT, PET, and SPECT. In addition, computational techniques, including fast algorithms and utilization of graphics processor units (GPUs) are always an important aspect of the meeting. More recently, significant time has been devoted to utilizing deep learning techniques in image reconstruction, either directly from the scanner's data or to provide corrections and enhancements to the reconstructed image. Such post-reconstruction processing can take the form of artifact reduction (e.g., sampling distortions, metal artifacts, or attenuation correction), de-noising, or other enhancements.

Although this special section for the Journal of Medical Imaging (JMI) contains only a small sampling of the 120 presentations, it also shows the range of topics covered. The first paper, by Y. Gao et al., looks at the development of a series of tissue-specific texture priors in CT by machine-learning techniques, for the purpose of enriching the tissue textures in the context of dose reduction while increasing the CT diagnostic potential; these priors allow for the different textures found in lung, bone, fat, and muscle for chest imaging. The second paper, by W. Whiteley, W. K. Luk, and J. Gregor, looks at 3D PET image reconstruction using Deep Learning by transforming the raw data into a set of 2D sinograms, upon which several corrections are made using traditional methods, and then using three neural network segments to (i) compress the data; (ii) invert the compressed data into an image; and (iii) apply scaling and denoising to obtain the final image. The third paper, by F. Terzioglu, derives and validates an exact inversion of Compton-camera data into image space; the technique maps the integral transform from the raw data to the Radon transform, which can then be directly inverted. The fourth paper, by V. Panin and S. Matej, looks at Fourier-based techniques for reducing rebinning and interpolation errors in space-based techniques for analytic time-of-flight (TOF) PET reconstruction; the technique is compared against commercially deployed software and can also be utilized in a non-TOF algorithm.

We would like to thank the many cooperative participants, who submitted very high quality abstracts and made excellent presentations. We especially thank the authors who contributed to this JMI special section.

\footnotetext{
*Address all correspondence to Scott. D. Metzler, E-mail: metzler@upenn.edu; Samuel Matej, E-mail: matej@pennmedicine.upenn.edu; J. Webster Stayman, E-mail: web.stayman@jhu.edu

() 2020 Society of Photo-Optical Instrumentation Engineers (SPIE)
} 